\title{
Nature of and lessons learned from Lunar lce Cube and the first deep space cubesat 'cluster'
}

Pamela Clark, Robert MacDowall, William Farrell, Cliff Brambora, Al Lunsford, et al.

Pamela Clark, Robert MacDowall, William Farrell, Cliff Brambora, Al Lunsford, Terry Hurford, David Folta, Benjamin Malphrus, Matt Grubb, Sarah Wilzcewski, Emily Bujold, "Nature of and lessons learned from Lunar Ice Cube and the first deep space cubesat 'cluster'," Proc. SPIE 10769, CubeSats and NanoSats for Remote Sensing II, 107690G (26 October 2018); doi: $10.1117 / 12.2320055$

EDIE Event: SPIE Optical Engineering + Applications, 2018, San Diego, California, United States 


\title{
Nature of and Lessons Learned from Lunar Ice Cube and the First Deep Space Cubesat 'Cluster'
}

\author{
Pamela Clark*a, Robert MacDowall ${ }^{\mathrm{b}}$, William Farrell ${ }^{\mathrm{b}}$, Cliff Brambora ${ }^{\mathrm{b}}$, Al Lunsford ${ }^{\mathrm{b}}$, Terry \\ Hurford $^{\mathrm{b}}$, David Folta ${ }^{\mathrm{b}}$, Benjamin Malphrus ${ }^{\mathrm{c}}$, Matt Grubb ${ }^{\mathrm{e}}$, Sarah Wilzcewski**a, Emily Bujold ${ }^{* * a}$ \\ a Jet Propulsion Laboratory, California Institute of Technology, 4800 Oak Grove Drive, Pasadena, \\ CA 91109; ' NASA Goddard Space Flight Center, 8800 Greenbelt Road, Greenbelt, MD 20771; \\ ${ }^{\mathrm{c}}$ Morehead State University, Space Science Center, Morehead, KY 40351; ${ }^{\mathrm{d}}$ NASA IV\&V, \\ Fairmont, WV 26554
}

*Pamela.E.Clark@jpl.nasa.gov; phone 1818 393-3262; fax 1818 354-8887; jpl.nasa.gov

** Jet Propulsion Laboratory, California Institute of Technology, Student Interns from Morehead State University

\begin{abstract}
Cubesats operating in deep space face challenges Earth-orbiting cubesats do not. 15 deep space cubesat 'prototypes' will be launched over the next two years including the two MarCO cubesats, the 2018 demonstration of dual communication system at Mars, and the 13 diverse cubesats being deployed from the SLS EM1 mission within the next two years. Three of the EM1 cubesat missions, including the first deep space cubesat 'cluster', will be lunar orbiters with remote sensing instruments for lunar surface/regolith measurements. These include: Lunar Ice Cube, with its 1-4 micron broadband IR spectrometer, BIRCHES, to determine volatile distribution as a function of time of day; Lunar Flashlight, to confirm the presence of surface ice at the lunar poles, utilizing an active source (laser), and looking for absorption features in the returning signal; and LunaH-Map to characterize ice at or below the surface at the poles with a compact neutron spectrometer. In addition, the BIRCHES instrument on Lunar Ice Cube will provide the first demonstration of a microcryocooler (AIM/IRIS) in deep space. Although not originally required to do so, all will be delivering science data to the Planetary Data System, the first formal archiving effort for cubesats. 4 of the 20 recently NASA-sponsored (PSDS3) study groups for deep space cubesat/smallsat mission concepts were lunar mission concepts, most involving 12U cubesats. NASA SIMPLEX 2/SALMON 3 AO will create ongoing opportunities for low-cost missions as 'rides' on government space program or private sector vehicles as these become available.
\end{abstract}

Keywords: Moon, cubesats, volatiles, Broadband IR, Neutrons, Laser, lunarcubes, lunar orbiters, 6U, EM1

\section{LUNAR CUBESATS BEYOND LEO}

Unlike their earth-orbiting predecessors, deep space cubesats are required to have the full functionality, and active control systems, of any spacecraft operating in deep space. $156 \mathrm{U}$ cubesats with diverse payloads entering deep space over the next two years have been (MarCO) or are being built (EM1 13), effectively 'prototypes' for deep space cubesats. Three of them, Lunar Ice Cube, Lunar Flashlight, and LunaH-Map, are science requirements-driven lunar orbiters with the goal of increasing our knowledge of lunar volatiles, acting as the first de facto deep space cubesat cluster.

Out of the 13 cubesats to be deployed by EM1 (Table 1) sometime during 2020, 8 are specifically designed for lunar or cislunar operation: Lunar Ice Cube, Lunar Flashlight, and LunaH Map, all of which will be described in more detail below. LunIR is a Lockheed Martin flyby which will perform IR thermography and demonstrate a new propulsion system. Omotenashi is a JAXA-sponsored semi-hard impactor and radiation environment monitor. NASA CubeQuest challenge selectees Team Miles (Tampa HackerSpace), CUE3 (U Colorado), and Cislunar Explorers (Cornell) will demonstrate communication and propulsion technologies in cislunar space. The compact Ion Analyzer and energetic neutral imagers instruments of the proposed Hydrogen Albedo Lunar Orbiter (HALO) received further development funds, through the NASA SIMPLEx program, and compact surface instruments were proposed for NASA's Development of Advanced Lunar Instruments (DALI) program, to be selected later in 2018. Meanwhile, two 12U cubesat missions, LUMIO (Meteoroid 
Impact Detection from L2) and VMMO (Volatiles/Mineralogy Shackleton Crater Orbiter) were selected for the ESA LUCE program (Table 1).

\section{Table 1: Planned Cislunar Missions}

\begin{tabular}{|l|l|l|}
\hline Mission & Goal & Program \\
\hline Lunar Ice Cube & Orbit, any form or state of water, as function of time of day & NASA HEOMD \\
\hline Lunar Flashlight & Orbit, South Pole surface ice in PSRs & NASA HEOMD \\
\hline LunaH-Map & Orbit, South Pole, ice on surface to 1 meter depth & NASA/SMD \\
\hline LunIR & flyby, lunar regolith properties & NASA HEOMD \\
\hline Omotenashi & impactor, radiation & JAXA (on EM!) \\
\hline Team Miles & CubeQuest, demonstrate propulsion and communication milestones & NASA STMD \\
\hline CUE3 & CubeQuest, demonstrate propulsion and communication milestones & NASA STMD \\
\hline Cislunar Explorers & CubeQuest, demonstrate propulsion and communication milestones & NASA STMD \\
\hline LUMIO & L2 orbit, camera, meteoroids impacting Moon from L2 & ESA LUCE \\
\hline VMMO & Orbit, Shackleton, volatiles, radiation & ESA LUCE \\
\hline
\end{tabular}

Several recent NASA Planetary Science Deep Space SmallSat Studies (PSDS3) have proposed lunar smallsat missions, not cubesats as the concepts were based on conventional spacecraft subsystems including landers. The payloads in most cases would be compatible with the $12 \mathrm{U}$ cubesat paradigm if the teams were willing to embrace cubesat subsystems as yet untested in deep space and be deployed as packages on provided landers and/or orbiters. These include:

- Mini-Lunar Volatiles (MiLuV) (PI Petro, GSFC). Payload: Lunar Ice LIDAR spectrometer measuring at 1.6 and 3 microns in lunar orbit [1]

- Bi-Satellite Observations of the Lunar Atmosphere above Swirls (BOLAS) (PI, Stubbs, NASA GSFC) Payload: Ion Spectrometer, Energetic Neutral Atom Imager, Mini-magnetometer, Plasma Wave System. Tethered satellites at two altitudes [2]

- Irregular Mare Patch Exploration Lander (IMPEL) (PI Draper, NASA JSC) Payload: Camera System on Small Lander [3]

- Lunar Water assessment, transportation, and resource potential (WATER) (PI Hibbitts APL) Lunar water assessment, transportation, and resource. Payload: Multi-spectral IR laser and Mid-IR Imager, and Neutron Spectrometer in polar periapsis orbit [4]

- Cubesat X-ray Telescope (CUBEX) (PI Romaine SAO) Payload: X-ray Imaging spectrometer and Solar X-ray monitor to determine lunar surface elemental abundances from circular $6000 \mathrm{~km}$ polar orbit [5]

\section{INTERNATIONAL AND COMMERCIAL CONTEXT}

As Low Earth Orbit, the previous domain of cubesats, has become a virtually financially achievable and sustainable venue, attention has turned to cislunar space as the next 'conquerable frontier'. Below is summary of national space program planned activities and their implications for cubesats:

- USA: recent lunar initiative supporting the development of lunar payloads and commercial landers and orbiters to carry these payloads to the Moon. Low cost payloads in standardized deployers especially favored. Ongoing development of launch vehicle (SLS), capsule, and lander, and plan to develop lunar orbiting station, for human crew who could deploy cubesats in orbit or on the surface.

- China: plan for long-term cislunar industrial development and space-based power. Cubesats could be used for high fidelity local or orbital reconnaissance. 
- Russia: plan for lunar polar lander, orbiter, sample return, and joint (with USA) orbiting spaceport.cubesats candidates for human or robotically deployable packages, interior or exterior environmental monitors. Enthusiasm for lander science and gateway payloads shown at recent NASA-sponsored workshops.

- Europe: Collaboration with Russians and Chinese on International Moon Village. Landers or rovers with compact, deployable payloads to characterize, monitor highly desirable.

- Japan, India: plan for lander, rover, sample return, tech demos in support of long-term base. Human or robotically deployable packages here also.

- Commercial Interests: developing lunar destination launch services, landers, and orbiters to start as early as 2019. These include: Astrobotic/Spacex, MoonEx/Rocketlabs, Masten/ULA. Compact, low cost payloads, easily integratable and deployable most desirable.
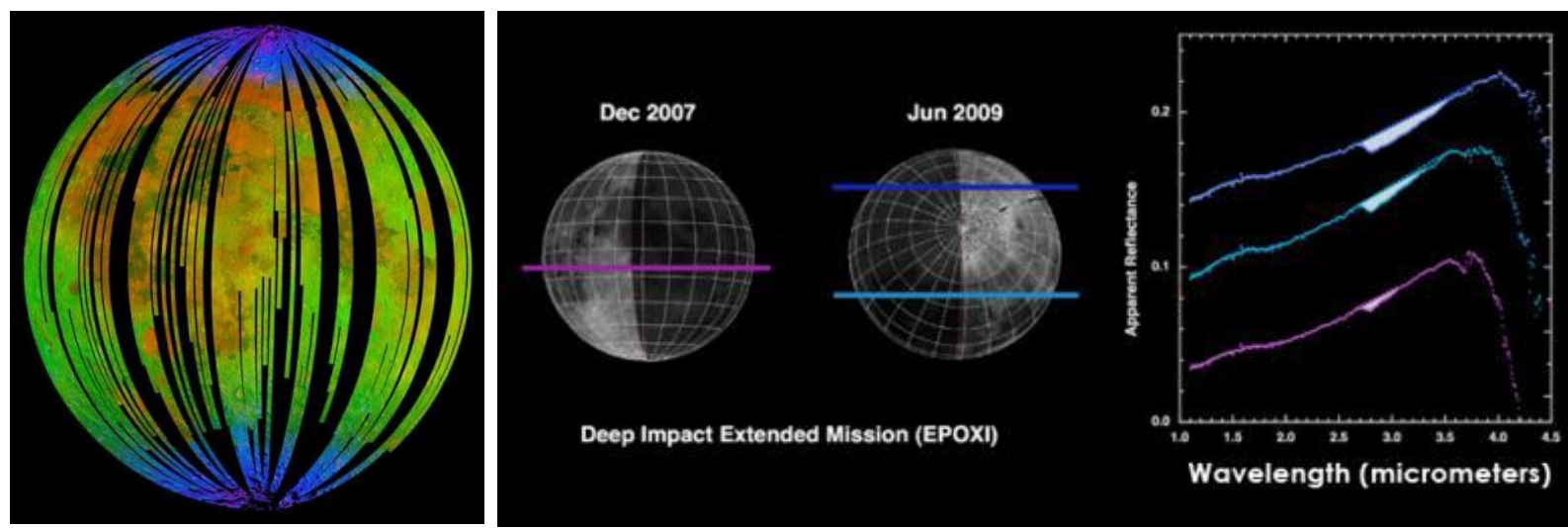

Figure 1: Water and hydroxyl on the moon. Left: indicated by blue, deep orange, deep green. Credit: ISRO/NASA/JPL Chandrayaan-1 Mission. Right: Absorption feature depth (abundance) correlated with time of day. Credit: NASA/JPL/Caltech/UMD Deep Impact Mission.

\section{SCIENTIFIC CONTEXT}

New discoveries as well as trends over the last decade have caused increasing interest in the Moon.

Findings from several lunar missions nearly a decade ago played a major role in modifying our understanding of the role and potential availability of resources on the Moon. The Lunar Crater Observation Sensing Satellite (LCROSS) observed abundant water and ice released from the floor of polar crater Cabeus at impact [6,7]. Unexpectedly, an $\mathrm{OH}$ feature, indicating the presence of an $\mathrm{OH}$ veneer extending from poles to equator, was derived from IR spectral absorption features detected by instruments onboard Chandrayaan (Figure 1), Deep Impact (Figure 1), and Cassini [8,9,10]. These findings suggest that the Moon has both water and $\mathrm{OH}$ diurnal cycles that are global and not yet fully verified and understood. Furthermore, the apparent presence of water and $\mathrm{OH}$, even at mid-latitudes [11], and of water associated with pyroclastic deposits [12] has a profound effect on our understanding of the nature of surface chemistry on all airless exposed bodies. Specifically, if such $\mathrm{OH}$ and water is of solar wind origin, then the observations suggest that $\sim 1 \mathrm{keV}$ protons interacting with any oxide-rich surface will thus generate $\mathrm{OH}$ via surface interactions. In essence, the discovery of lunar $\mathrm{OH}$ at lunar latitudes as low as 30 degrees could imply that all exposed oxide-rich bodies are miniature water and $\mathrm{OH}$ factories, a finding with implications for In Situ Resource Utilization. Further reconnaissance of our nearest neighbor, the Moon, will be essential to determine the viability of such a proposition. SMD decadal survey. HEOMD SKGs. 


\section{LUNAR CUBESAT 'CLUSTER'}

The EM1 lunar orbiters Lunar Ice Cube, Lunar Flashlight, and LunaH-Map provide complimentary measurements essential in understanding water origin, cycle, and dynamics on the Moon, creating the first de facto deep space cubesat cluster, as illustrated in Table 2.

Table 2: Comparison of Three Lunar Orbiter Cubesat Cluster Missions

\begin{tabular}{|c|c|c|c|}
\hline Primary Mission Goal & Instrument & Measurement & Operation \\
\hline $\begin{array}{l}\text { Lunar Ice Cube: Determine } \\
\text { distribution of forms and } \\
\text { components of water as a } \\
\text { function of time of day in } \\
\text { order to provide inputs to } \\
\text { constrain models for global } \\
\text { water origin, production, and } \\
\text { loss }\end{array}$ & $\begin{array}{l}\text { Compact Broadband } \\
\text { IR (1 to } 4 \text { microns to } \\
\text { completely capture } \\
\text { broad } 3 \text { micron band }) \\
\text { point spectrometer } \\
\text { with up to } 10 \mathrm{~nm} \\
\text { spectral resolution. }\end{array}$ & $\begin{array}{l}\text { IR reflectance with wavelength- } \\
\text { dependent water components and } \\
\text { form absorption features, with } \\
\text { detection for }>\mathbf{1 0 0} \mathbf{~ p p m} \text { and }>/=\mathbf{1 0} \mathbf{~ k m} \\
\text { spatial resolution. }\end{array}$ & $\begin{array}{l}\text { Nearly polar highly elliptical } \\
\text { equatorial periapsis ( } 100 \\
\text { km)orbits ( } 7 \text { hour period) with } \\
\text { repeating coverage once a } \\
\text { lunar cycle for up to } 6 \text { lunar } \\
\text { cycles providing varying time } \\
\text { of day coverage for } \sim \mathbf{1 0 \%} \text { of } \\
\text { lunar surface across all } \\
\text { latitudes. }\end{array}$ \\
\hline $\begin{array}{l}\text { LunaH Map: Determine } \\
\text { distribution of surface and } \\
\text { subsurface }(<1 \mathrm{~m}) \text { ice }\end{array}$ & $\begin{array}{l}\text { Compact Neutron and } \\
\text { Gamma-ray } \\
\text { spectrometer }\end{array}$ & $\begin{array}{l}\text { Decrease in epithermal neutron flux } \\
\text { (for }>/=20 \% \text { decrease) associated } \\
\text { with protons (ice)) (to 10's of } \mathbf{c m} \\
\text { depth) at poles with } 7.5 \mathrm{~km} \text { spatial } \\
\text { resolution within }<2 \text { degrees of poles. }\end{array}$ & $\begin{array}{l}\text { Elliptical south polar periapsis } \\
(>10 \mathrm{~km}) \text { orbits }(10 \text { hour } \\
\text { period) greatly overlapping at } \\
\text { poles, } 2 \text { months. }\end{array}$ \\
\hline $\begin{array}{l}\text { Lunar Flashlight: Determine } \\
\text { distribution of ice at surface } \\
\text { in permanently shadowed } \\
\text { regions at poles }\end{array}$ & $\begin{array}{l}\text { optical receiver aligned } \\
\text { with lasers emitting at } \\
\text { wavelengths associated } \\
\text { with water ice } \\
\text { absorption and } \\
\text { continuum. }\end{array}$ & $\begin{array}{l}\text { Ratio continuum and absorption } \\
\text { reflectance bands to quantify surface } \\
\text { ice abundance in permanently } \\
\text { shadowed areas at poles for }>0.5 \\
\text { wt. } \% \text { with } 1 \mathbf{k m} \text { spatial resolution } \\
\text { within } 10 \text { degrees of poles. }\end{array}$ & $\begin{array}{l}\text { Elliptical south polar periapsis } \\
(\sim 14 \mathrm{~km}) \text { orbits ( } 5 \text { day) greatly } \\
\text { overlapping at poles, } 2 \text { months. }\end{array}$ \\
\hline
\end{tabular}

\section{Lunar Ice Cube (LIC)}

The Lunar Ice Cube Mission goal is the determination of abundances of forms and components of water (e.g., adsorbed water, bound water, ice, hydroxyl). as a function of time of day.

BIRCHES (Figure 2) [13] (Broadband IR Compact High-resolution Exploration Spectrometer), the Lunar Ice Cube payload, is a broadband, flexible, cubesat-scale IR spectrometer concept, flyable as a point or imaging spectrometer as bandwidth allows (point spectrometer for this application due to bandwidth limitations). The instrument will provide hyperspectral measurements of volatile-related features in the 1 to 4 micron region, especially the so-called ' 3 micron band', consisting of several water-related features. Previous lunar orbiting Near IR spectrometers did not provide measurements beyond 3 microns. BIRCHES has heritage from OSIRIS-REx (Origins Spectral Interpretation Resource Identification and Security-Regolith Explorer) OVIRS (OSIRIS-REx Visible InfraRed Spectrometer) and New Horizons LEISA (Linear Etalon Imaging Spectral Array) $[\mathbf{1 4 , 1 5 , 1 6}$. BIRCHES represents a compact version of a family of simple, versatile, and low-cost instruments, adapted as OSIRIS Rex OVIRS is adapted, from the GSFC-developed LEISA (Ralph) $[14,15,16,17]$. LEISA-derived instruments represent (lower cost, mass, volume, power) concepts in spectrometer design made possible by large-format infrared detectors combined with advances in thin-film technology in the form of nondispersive thin film filters, called wedged or linear variable etalon filters (LVE or LVF), as wavelength selection elements. Such spectrometers represent a great reduction in optical and mechanical complexity and volume compared to conventional grating, prism, or Fourier transform spectrometers and mechanically or electrically tunable filter systems. To meet requirements while maintaining a low-cost profile, BIRCHES utilizes:

- Teledyne H1RG Focal Plane Array with a linear variable filter, spares from OVIRS.

- Thermally isolated, compact optics, designed and developed as GSFC internal R\&D 
- AIM Microcryocooler developed for AFRL being qualified on Lunar Ice Cube for deep space

- H1RG generic compact detector interface electronics with flexible architecture suitable for point or imaging spectrometers leveraging ongoing NASA/GSFC internal R\&D efforts to developed mixed signal ASICs.
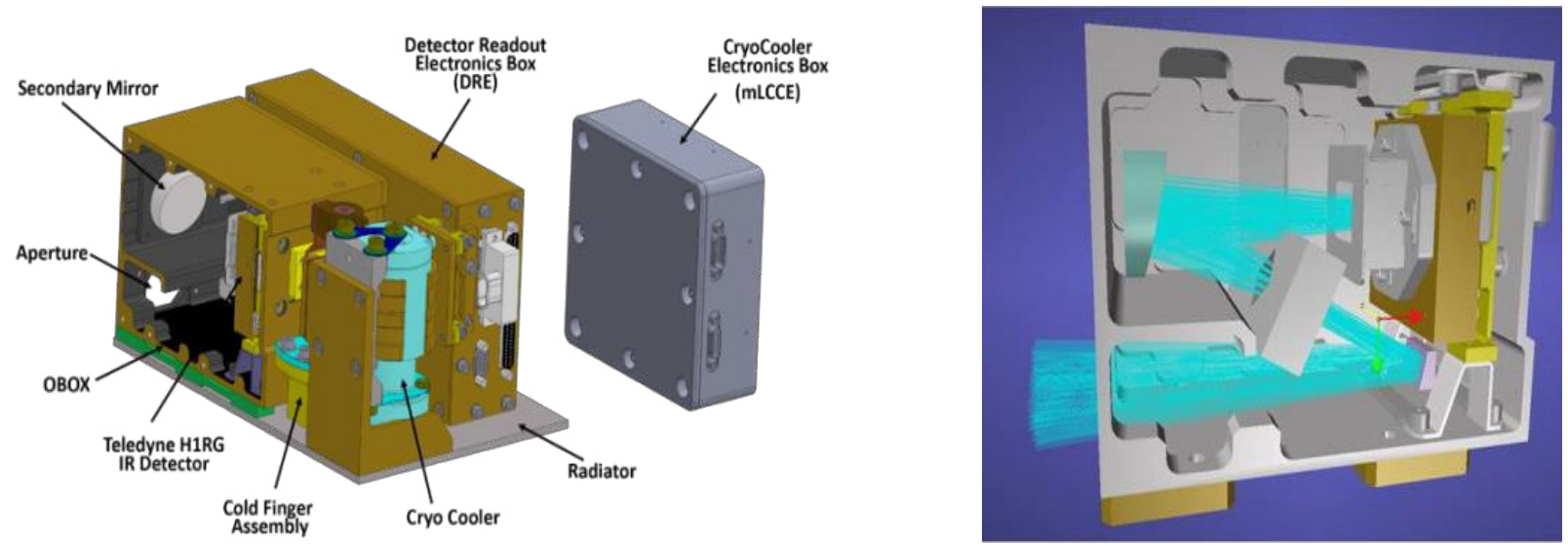

Figure 2: Lunar Ice Cube BIRCHES spectrometer (above) with ray tracing (below) with components as described in text.

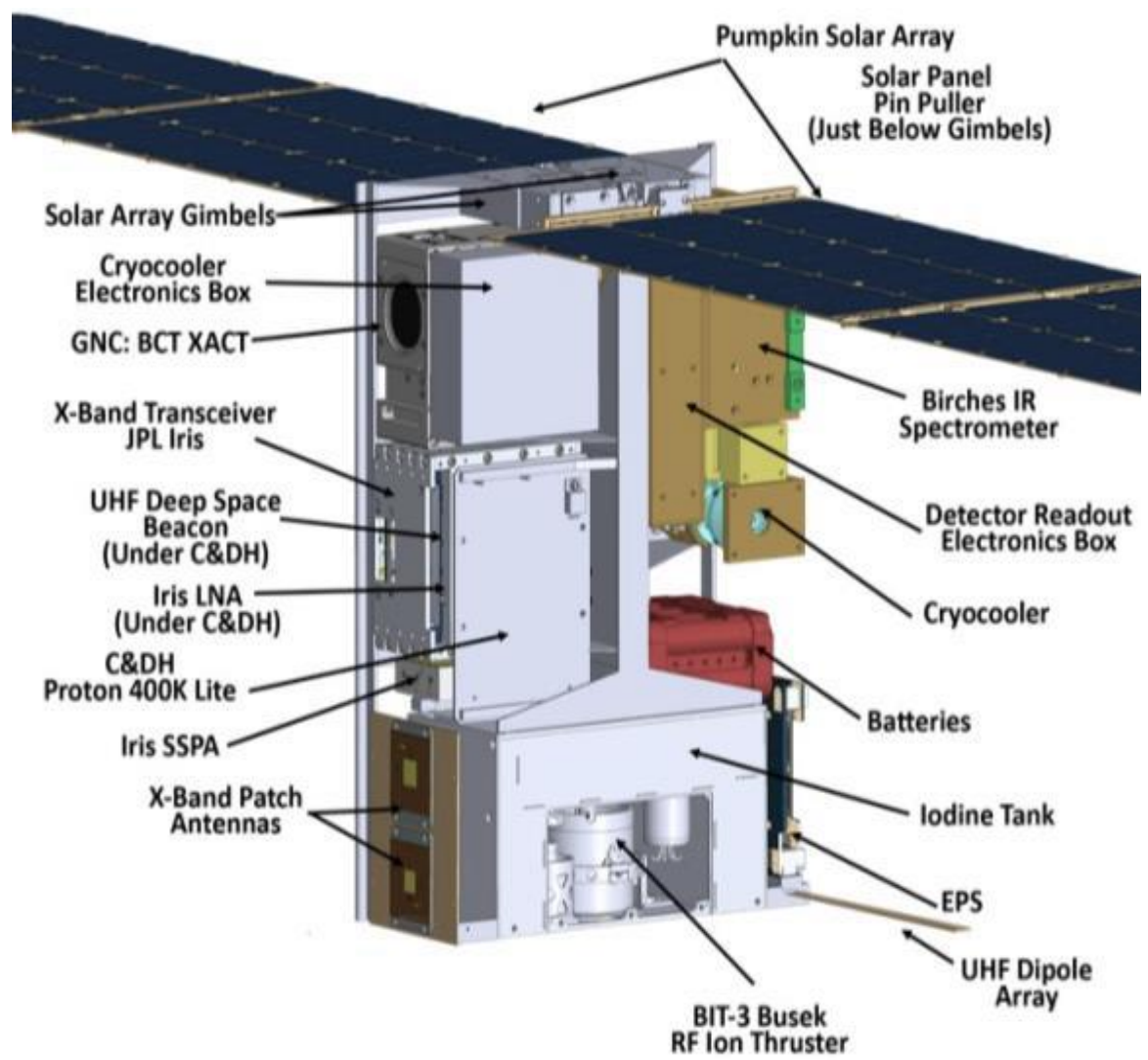

Figure 3: Lunar Ice Cube 6U spacecraft. Subsystems described in text. Note BIRCHES spectrometer and Busek propulsion system, both tech demos for this mission. 
Major components of the BIRCHES instrument include [13]:

- an existing (at GSFC) Teledyne H1RG Mercury Cadmium Telluride (HgCdTe) $18 \mu \mathrm{m}$ pixel (pitch) 2D focal point array (FPA) with associated Linear Variable Filter (LVF) originally built as spares for OSIRIS Rex OVIRS (Figure 6) [1]. OVIRS uses a $512 \times 512$ pixel region of a 1024 x 1024 FPA. The H1RG arrays are constructed by hybridizing (via Indium bump bonds) a photosensitive $\mathrm{HgCdTe}$ detector layer, with the appropriate $\mathrm{Hg} / \mathrm{Cd}$ ratio, to a CMOS Read Out Integrated Circuit (ROIC). The array substrate is removed, allowing photon sensitivity over the entire range and also reducing the effect of spurious signals from cosmic rays.

- The LVF, a proven and compact means of line separation, an etalon, deposited on one surface as a substrate as described [20]. Etalon thickness and therefore transmitted wavelength varies as a function of position. BIRCHES utilizes three (out of the five provided by OVIRS) 102 x 512 pixel wedged filter segments bound together in a single assembly and mounted within $1 \mathrm{~mm}$ of the FPA surface. Because the wavelength may vary somewhat along a column, only those pixels in each column lying near the central wavelength of appropriate spectral range will be summed. The central wavelength of each pixel will be determined during ground calibration. Resolving power and resolution vary as a function of wavelength. Resolution is $10 \mathrm{~nm}$ and resolving power 350 between 2.9 and $3.6 \mu \mathrm{m}$, surrounding the critical $3 \mu \mathrm{m}$ band, well within the state-of-the art.

- optics box with an uncomplicated design, consisting of lightweight optically coated all magnesium components including two off-axis mirrors, separated by an adjustable field stop allowing change the footprint dimension by an order of magnitude, to adjust for variations in altitude and/or incoming signal. The optics have a dedicated radiator to maintain a $<220 \mathrm{~K}$ environment for the optics during data taking.

- The IRIS/AIM SX030 [2] single piston linear cooler with its compact and efficient miniaturized low cost microcryocooler electronics already developed for the US Air Force. It has improved cooling capacity (0.6W @ 140K), a moderately larger profile with the cryocooler electronics, cryocooler pump, and separate cold finger assembly, but far less jitter and a far more radiation tolerant controller than the RICOR 562S we originally considered. IRIS/AIM SX030 [2] and controller will meet our requirement to maintain the detector at $<115 \pm 1 \mathrm{~K}$ using from 5 to $10 \mathrm{~W}$ of onboard power.

- Compact generic DRE, designed to be easily reconfigurable for point or imaging spectrometer for the family of Teledyne HCT HnRG spectrometers, combined with heritage from our ongoing IRAD work on low cost, compact electronics, to meet the performance and compact volume requirements. Both of these are challenging requirements primarily due to the compact nature of the design. The DRE features a dual channel commercially available ADC converter and a PRO-ASIC-III FPGA to stimulate the detector with the appropriate biases and clocking schemes, digitize up to two outputs from the detector, gather the samples from the A/D converters, collect them, average them as required to reduce the data rate to better match the expected down link allocation $(\sim 128 \mathrm{kbps})$, and format the results into a packet format for transmission off the board

- The 6U spacecraft (Figure 3) utilizes and demonstrates the functionality for deep space of the Busek High ISP RF Ion Engine for propulsion, the BCT attitude knowledge and control system (XACT Star Tracker, Reaction Wheels, IMU), version 2.1 of the JPL Iris ranging transceiver, and the inexpensive Space Micro flight computer, designed to be radiation tolerant. More details. LunaH Map will also fly the same Busek engine, and both LunaH Map and Lunar Flashlight the Version 2.1 Iris communication subsystem.

Lunar Ice Cube will fly in a nearly polar, highly elliptical, equatorial periapsis orbit designed to provide repeat coverage of the same orbital swaths systematically at varying times of day during up to six consecutive lunar cycles (Figure 4). In this way, such overlapping coverage will be provided for up to $10 \%$ of the lunar surface across all latitudes. 


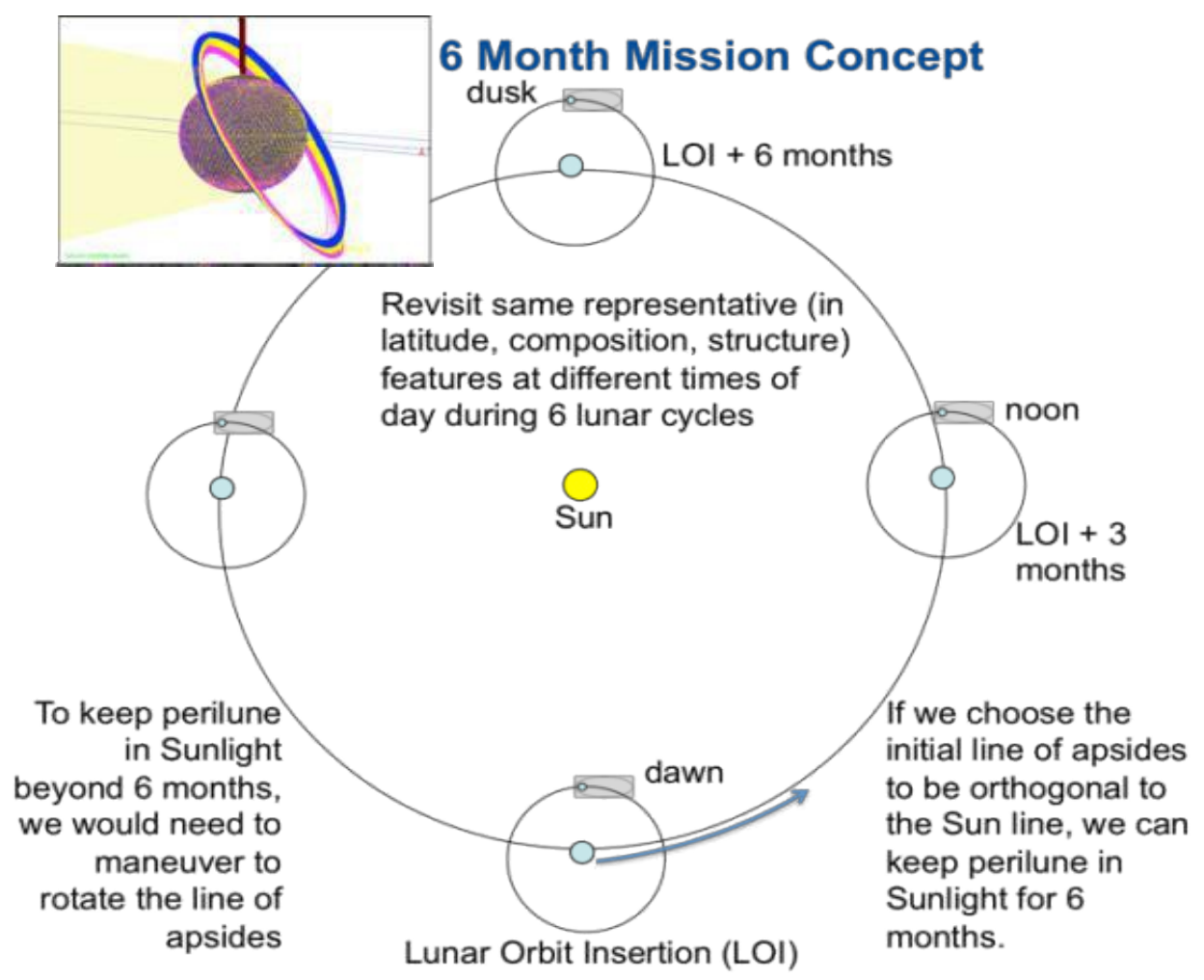

Figure 4: Lunar Ice Cube Mission Concept providing coverage from same orbital swaths at different times of day as Earth-Moon system revolves around the sun.

\section{LunaH-Map (LM) and Lunar Flashlight (LF)}

LunaH-Map [18] (Figure 5) and Lunar Flashlight [19] (Figure 6), both 6U cubesats, are in very elliptical, long period (10 hours for LunaH Map, 5 days for Lunar Flashlight), low ( $<10 \mathrm{~km}$ for LunaH Map, $14 \mathrm{~km}$ for Lunar Flashlight), south polar periapsis orbits for 2 months to search for evidence of ice in 'cold traps' at the poles.

LunaH Map will search within two degrees of the poles for surface or subsurface (down to 1 meter) ice with a resolution of $7.5 \mathrm{~km}$. Its two compact neutron spectrometers, utilizing a new material (CYCL) for neutron detection which has distinct advantages over He3 conventionally used [20]. CYCL (elpasolite), a new scintillator material, is sensitive to both neutrons and gamma-rays (which give differently shaped light pulses at different energies and thus are separable) and has a higher efficiency than $\mathrm{He} 3$ in the energy range of interest. It can be grown in a variety of shapes and sizes. Though not used before for neutron detection from orbit, CYCL has been demonstrated under lunar environmental conditions. Neutron spectrometers are designed to observe suppression in neutron flux (low epithermal neutron flux) indicating the presence of protons within 1 meter of the surface. Such detections, associated with permanently shadowed regions or burials from recent impact events, are likely to be associated with ice as opposed to solar wind (proton) implantations at the (illuminated) surface.

Lunar Flashlight will observe permanently shadowed areas within ten degrees of the poles with a spatial resolution of 1 $\mathrm{km}$. Lunar Flashlight utilizes near IR lasers emitting at wavelengths associated with water ice $(1.495+/-0.020$ and 1.85 $+0.020 /-0.030$ microns) peak absorption and the nearby reflectance continuum $(1.064+/-0.020 \mathrm{~nm}$ and $1.99+0.025 /-$ 0.020 microns) with a full wide at half $\max$ of $<20 \mathrm{~nm}$ [??]. Actively illuminating the surface at nadir, the laser 'transmitters' are aligned with optical receivers (InGaAs near IR detectors) which collect and measure a portion of the signal reflected from the surface, designed to minimize stray light and to maximize reflected signal detection with an optimal focal length and a field of view that allows for anticipated beam divergence. Decrease in the returning 1.04/1.50 or 1.85/1.99 signal ratios indicates an absorption feature and thus the presence of surface ice. Background measurements are acquired with the lasers off. 


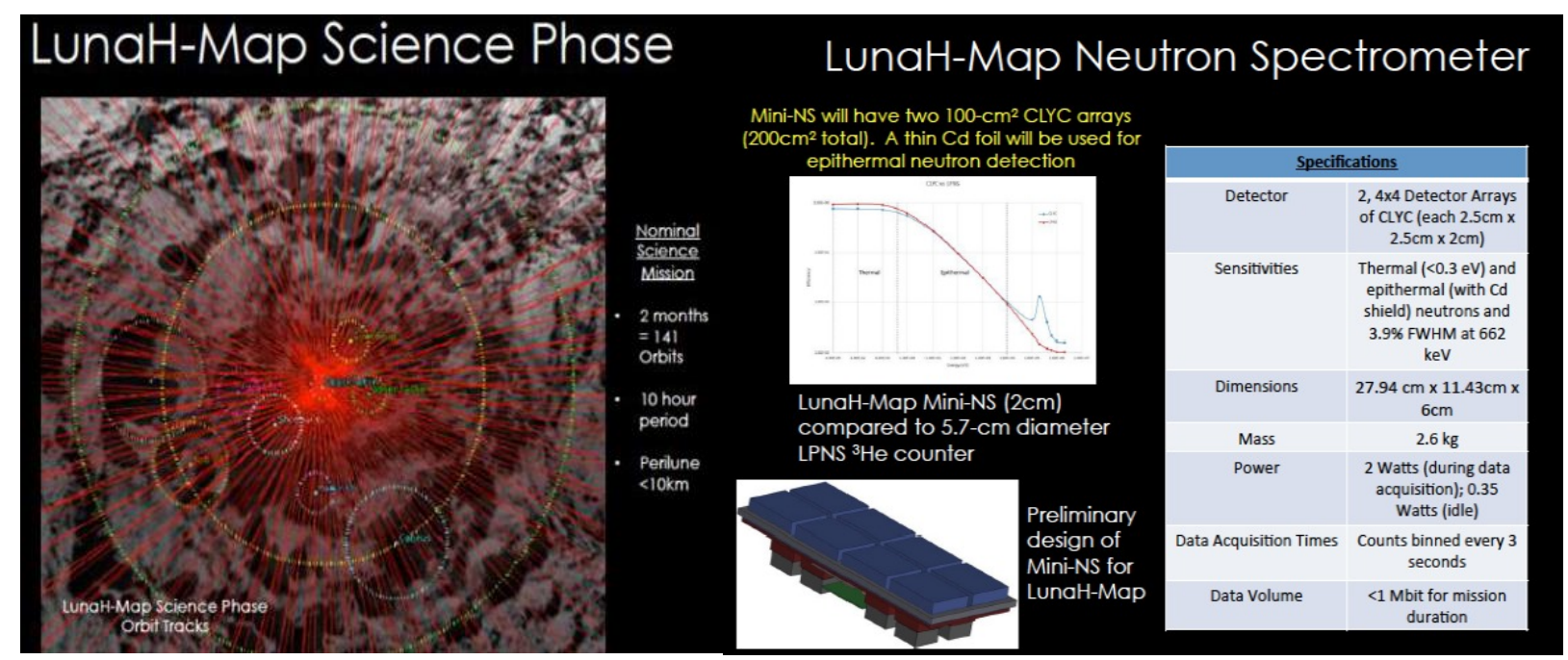

Figure 5: LunaH-Map science measurement coverage for south pole as described in Table 2 (left) and characteristics of Neutron Spectrometer (right). [17, 2017 LEAG presentation online]

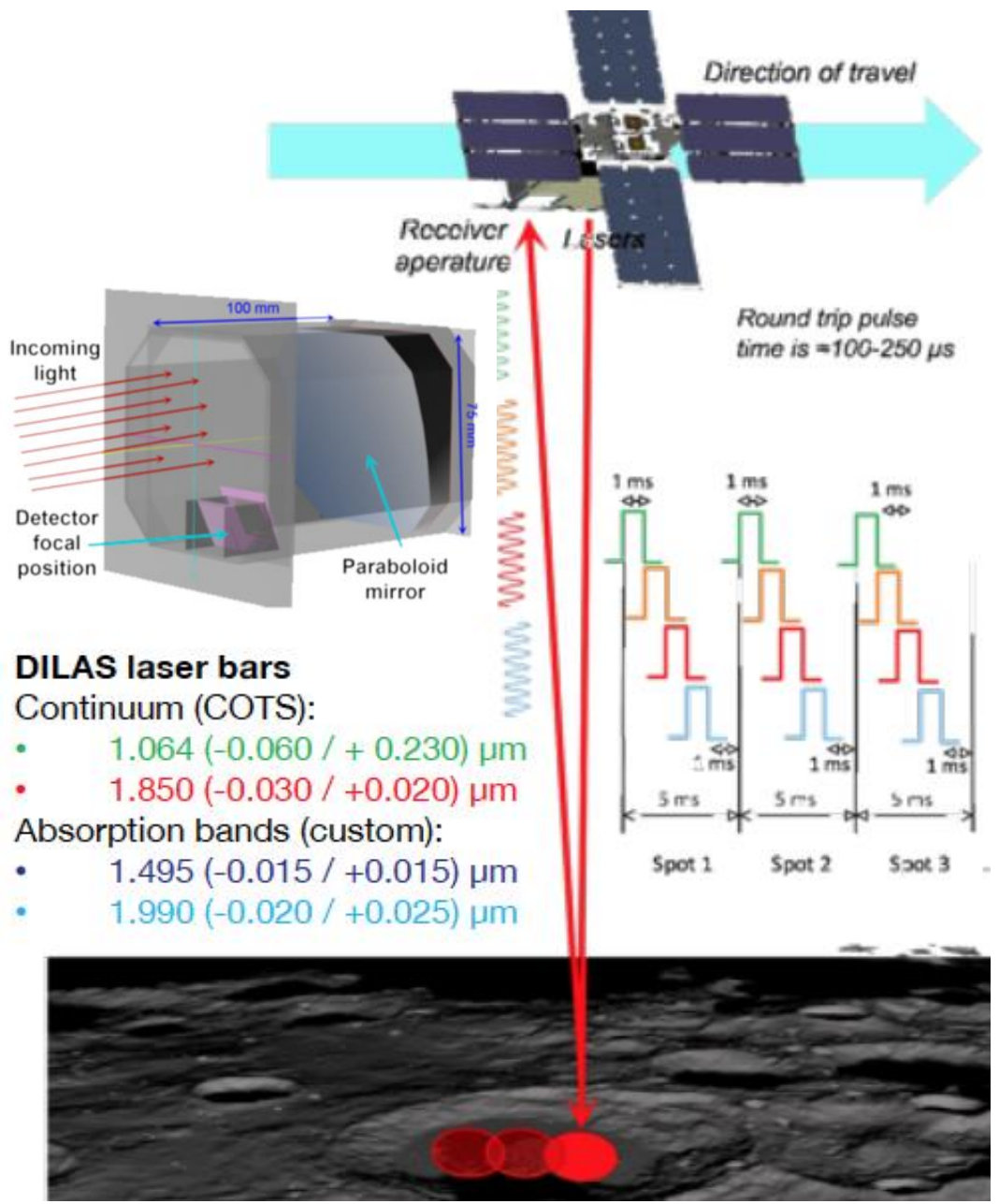

Figure 6: Lunar Flashlight Mission concept illustrating nature and operation of laser described in text as well as receiver. (Insert A). [21, 2017, Cubesat Science Symposium presentation online. 


\section{Cluster Contributions to Understanding the Water Cycle}

Each of the missions contributes to our understanding of the lunar global water cycle, and its impact on in situ surface modification processes (degree of interaction with solar wind protons resulting in physical or chemical changes on grain surfaces and the release or generation of volatiles) and on the potential generation or storage of water as a resource. Table 3 describes the impact of each mission on our understanding of sources and sinks for water.

Table 3: Comparison of Volatile Missions Impacts on understanding the water cycle

\begin{tabular}{|l|l|l|l|l|l|}
\hline $\begin{array}{l}\text { Water } \\
\text { source } \\
\text { or sink }\end{array}$ & $\begin{array}{l}\text { Solar Wind (time of } \\
\text { day, latitude, } \\
\text { shadowing effects) }\end{array}$ & $\begin{array}{l}\text { Impact } \\
\text { (transients) }\end{array}$ & $\begin{array}{l}\text { Pyroclastics, other bound- } \\
\text { water minerals (globally Cold Traps } \\
\text { distributed volcanic deposits) }\end{array}$ & $\begin{array}{l}\text { Thermal Migration } \\
\text { (shadowed } \\
\text { (time ogions, buried ice) } \\
\text { poleward facing slopes }\end{array}$ \\
\hline LIC & Time of day & maybe & yes & no & yes \\
\hline LM & shadowing & maybe & no & yes & no \\
\hline LF & shadowing & maybe & no & yes & no \\
\hline
\end{tabular}

\section{DATA MANAGEMENT STRATEGY FOR LOW-COST 'CLUSTER' MISSIONS}

The missions of the first deep space cubesat cluster have limited budgets for data management but are required to deliver data to a formal archive, the Planetary Data System (PDS) (Figure 7). JPL Advanced Multi-Mission Operations System/Multi-Mission Ground System (AMMOS/MMGS) has made a light version of mission operations tools (AIT) for extracting instrument measurements and engineering data from telemetry. Individual missions must then write their own code to produce data products. Lunar Ice Cube has used python-based code for producing raw or level 0 data, representing the readout from the BIRCHES focal plane array, to create Experiment Data Records. Other tools developed to for the streamlined AMMOS PDS Pipeline Service (APPS) include label design and verification, bundling into agreed upon archive structure, PDS delivery, and report generation. Each mission must write data product, archive structure, and software interface specification documents, though a simplified template has been developed by Lunar Ice Cube. To produce level 1 and above data products (calibrated spectra, water-related feature maps), Lunar Ice Cube will leverage algorithms developed for the OSIRIS REx OVIRS instrument, BIRCHES predecessor.

\section{NEXT STEP: THE LUNAR SURFACE}

Credible opportunities for delivery of small payloads to the lunar surface or lunar orbit via commercial landers are emerging in the coming decade. The latest scientific findings (See SCIENTIFIC CONTEXT) indicate the presence of water, a useful resource, in the regolith of Moon and other atmosphereless bodies. Further reconnaissance, thus more extensive in situ observation, is essential to establish whether water is an extractable resource. Characterization of the highly interactive volatile/field/energetic particle/dust environment of the lunar surface and subsurface, requires continuous (day/night, multiple cycle) operation. The challenges in achieving such goals, particularly utilizing low-cost, compact, deliverable packages, are described in CURRENT DRIVING REQUIREMENTS. Surface networks of stationary or mobile platform instruments such as Infrared (absorption feature) Imagers, energetic particle analyzers, mass spectrometers, electric field instruments or magnetometers, interior sounders, could certainly be envisioned as part of this network [22].

\section{CURRENT DRIVING REQUIREMENTS}

The Moon, from orbit or on the surface, is a challenging operational environment. Greater utilization of cislunar space will require an infrastructure we do not yet have in place. Driving requirements for operating in this environment [23] described below.

\section{Environmental Mitigation}

The temperature range varies from $400 \mathrm{~K}$ to $90 \mathrm{~K}$ at the equator to $150 \mathrm{~K}$ to $50 \mathrm{~K}$ in subpolar regions over each lunar cycle (28 days, except near the poles where days and nights can be much longer), with the most rapid changes at dawn and dusk 
LIC S/C BIRCHES

\section{Telemetry}

1) What does BIRCHES send to C\&DH? See DRE FPGA Spec describing Readout and CCSDS Packets

2) What does C\&DH send to Comm? See ICD Specifying Subsystem Packets Organization

3) What does Comm send to DSN? See IRIS Frames Specification and FGICD

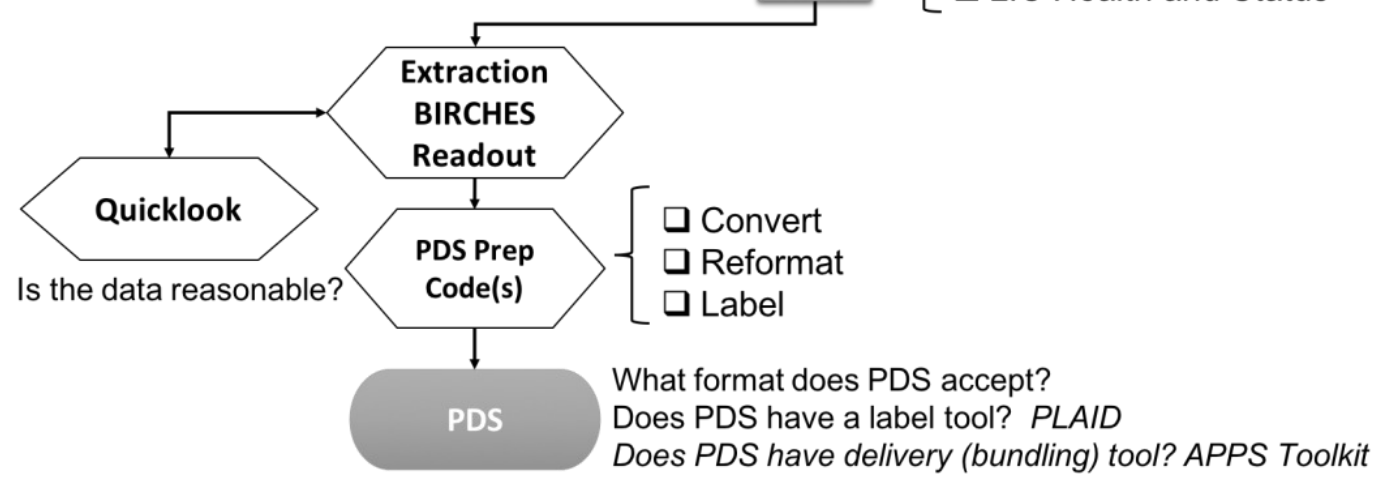

Figure 7: BIRCHES Data Flow from Lunar Ice Cube Spacecraft to the Planetary Data System, utilizing AIT Tools to extract BIRCHES focal plane array readout from frames, packet organizational structure, and BIRCHES CCSDS packets to produce labels and Experiment Data Records in ASCII CSV format, which are then delivered using APPS pipeline tools.

(terminator crossings) [12]. Thermal protection systems must consider not only the sun but thermal emission from the surface, which is covered with a regolith with very low thermal conductivity. This makes operating in low orbit or on the surface very challenging. Special radiators/reflector systems, based on those used on Apollo, have already been developed for this purpose. On the other hand, the biggest thermal challenge is operating, or even surviving, during lunar night with compact, low-cost surface payloads. The conventional approach, requiring radioisotopes, is relatively costly and inefficient.

In addition, movement of any kind on the surface will stir up lunar regolith particles at the surface, causing them to act as a contaminant on surrounding surfaces [13]. The behavior of lunar dust, described as 'abrasive Velcro', results from the extremely complex surface morphology of the elongated, interlocking, glassy shards, which, acting as insulators, utilizing the nooks and crannies as sources and sinks for charge. Mechanical brushing used in the Apollo program damaged the astronaut's spacesuits almost to the point of puncture. Proposed electrostatically-based approaches have not yet been attempted. Mechanisms that could come in contact with the regolith need to be protected, especially if longer term use is contemplated.

\section{Active Control Systems}

The Moon has numerous pronounced mass anomalies (mascons), and thus gravitational anomalies. These result from the infilling of the holes (craters) left by earlier large impact events with later stage volcanic material denser than the crust. Robust propulsion and attitude control systems are needed to support the station keeping, momentum dumping, and increased Delta $\mathrm{V}$ required to maintain most orbits. The exceptions are the polar, nearly circular, low altitude orbits called 'frozen orbits'.

\section{Communication}

Bandwidth is a limited resource in deep space, even in cislunar space. Mission and Program architectures that support dual communication capability (local and distant) on each spacecraft, communication 'hubs' or low-cost distributed and replaceable networks will be crucial to support the planned activity over the next decade. Smart onboard processing utilizing, for example, change detection algorithms for minimizing the downlink requirements, is highly desirable. 


\section{Transportation}

To date, though we have had many lunar missions, we haven't had an infrastructure that supports regular lunar visits. The success of the commercial sector at sending the first landers and orbiters to the Moon within the next two years as planned is crucial here. Astrobotic, Masten, and MoonEx seem the most likely candidates. Beyond that, the setting up of an efficient means of transportation between the Earth and Moon, perhaps through the use of cycling orbits as proposed by Buzz Aldrin, will be required.

\section{Mission Operations}

The utilization of standardized, compact, reconfigurable and/or deployable subsystems is essential to maintain the lowcost cubesat paradigm. Utilization of the Earth's magnetic or gravitational fields, the global positioning system, and the relatively benign environment in low Earth orbit minimized the need for active control systems and contributed greatly to the success of the cubesat paradigm. In deep space, in the absence of the Earth's magnetic and gravitational fields as well as communication network, cubesats require propulsion, orientation, thermal control, and more capable communication systems, resulting in greater operational complexity and demanding more reconfigurability. Because space and mass are limited, this reconfigurability typically increases operational complexity and must be achieved through the use of flight software. Thus, the cost of mission operations and flight software are not scalable downward in the same way as hardware development and launch costs for deep space cubesats. The development and utilization of standardized, shared, user friendly cubesat operating systems and mission operations tools will help to reduce these costs.

\section{LESSONS LEARNED}

The first deep space cubesat programs have provided some valuable lessons to date, even though not as yet completed [23]. Deep space cubesats have to face many more challenges than Earth-orbiting predecessors, and the science and/or technology requirements have been more stringent.

- The first deep space cubesats, being developed simultaneously, are prototypes, and costs can't be extrapolated directly from LEO cubesats built to date. even when a 'fudge factor' (like a friendly order of magnitude) is built in. Attempts to do so have so far resulted in underestimated cost. Earth Orbiting cubesats can utilize the Earth's magnetic and gravitational fields in lieu of active control systems (propulsion, attitude control), operate in a less extreme (thermal, radiation, lifetime) environment, and can utilize extensive and relatively close-at-hand ground-based antenna system or build a dedicated antenna relatively inexpensively. Costs for future deep space cubesats will be lower, and interpolatable, after these prototypes are developed and operated.

- The first deep space cubesats have been universally driven by more stringent, programmatically-set mission requirements, scientific and/or technological. Thus, the appropriate level of documentation and testing, and thus development paradigm and costs have yet to be established, though teams are currently experimenting with different approaches.

- As all working on the first generation deep space cubesats have discovered, costs aren't universally scalable based on size of payload or development model. Flight software development, mission operations, and science data management costs depend on mission complexity to a large extent, and deep space operations are intrinsically more complex than Earth operations.

- The harnessing by deep space missions of the cubesat paradigm, with rapid and lean development and operation, compactness, and standardization of deployment packaging and interfaces as touchstones, will require 1) sharing of personnel and equipment resources and tools, 2) automated, electronic capture systems for testing and documentation, and 3) a new track for deep space missions which capitalizes on emerging communication and transportation services, and prioritizes rapid technology qualification and/or reasonable cost multi-platform concepts. Further lessons and successful models for a deep space cubesat paradigm will emerge through the collaboration of those already using the cubesat paradigm to create an innovative and tailored solution with a standard platform for deep space missions. 


\section{ACKNOWLEDGMENTS}

This work was carried out at the Jet Propulsion Laboratory, California Institute of Technology, under contract with the National Aeronautics and Space Administration.

\section{REFERENCES}

[1] Petro, N., E. Mazarico, X. Sun, J. Abshire, G. Neumann, P. Lucey, "MiLuV Does it good - the Mini Lunar Volatiles Mission: A planetary science deep space smallsat study of a lunar orbiting mission" Lunar and Planetary Science 2018, LPI 2083, 2655.pdf, (2018)

[2] Stubbs, T., BOLAS

[3] Draper, D., J. Stopar, S. Lawrence, B. Denevi, K. John, L. Graham, J. Hamilton, Z. Fletcher, J. Gruener, S. Bertsch "The irregular mare patch exploration lander (IMPEL) smallsat mission concept", Lunar and Planetary Science 2018, LPI 2081, 1617.pdf (2018)

[4] Hibbitts, C., B. Clyde, D. Blewett, P Brandt, L. Burke, B. Cohen, J. Dankanich, D. Hurley, R. Klima, D. Lawrence, A. Mirantes, D. Moessner, W. Petterson, J. Plescia, J. Sunshine, J. Westlake "The Lunar WATER Mission: A PSDS3 feasibility study of a solar-electric propulsion small sat mission to characterize the water on the Moon" Lunar and Planetary Science 2018, LPI 2083, 1748.pdf (2018)

[5] Hong, J., S. Romaine, L. Nittler, I. Crawford, D. Kring, N. Petro, K. Gendreau, J. Mitchell, L. Wintermitz, R. Kraft, A. Kenter, G. Prigozhin, R. Masterson, J. Evans, K. Bonner, A. Clark, A. Dave, A. Dono-Perez, M. Ebert, A. Kashani, D. Larrabee, D. Mauro, S. Montez, J. Mueting, D. Nguyen, L. Plice, K. Ronzano, L. Shen, T. Snyder, J. Stupl, B. Wickizer, "Cubesat X-ray telescope (CubeX) for lunar elemental abundance mapping and millisecond X-ray pulsar navigation" Lunar and Planetary Science 2018, LPI 2083, 2793.pdf (2018)

[6] Colaprete, A., P. Schultz, J. Heldmann, D. Wooden, M. Shirley, K. Ennico, B. Hermalyn, W. Marshall, A. Ricco, R. Elphic, D. Goldstein, D. Summy, et al., "Detection of Water in the LCROSS Ejecta Plume", Science, 330, 463-468, 2010.

[7] Schultz, P., B. Hermalyn, A. Colaprete, K. Ennico, M. Shirley, W. Marshall, "The LCROSS cratering experiment" Science, 330, 6003, 468-472, 2010.

[8] Pieters C. J. Goswami, R. Clark, M. Annadurai, J. Boardman, B. Buratti, J.P. Combe, M. Dyar, R. Green, J. Head, C. Hibbits, M. Hicks, P. Isaacson, R. Klima, G. Kramer, S. Kumar, E. Livo, S. Lundeen, E. Malaret, T. McCord, J. Mustard, J. Nettles, N. Petro, C. Runyon, M. Staid, J. Sunshine, L. Taylor, S. Tompkins, P. Varanasi, "Character and spatial distribution of $\mathrm{OH} / \mathrm{H} 2 \mathrm{O}$ on the Moon seen by M3 on Chandrayaan-1", Science, 326, 568-572, 2009.

[9] Sunshine, J. M., T. L. Farnham, L. M. Feaga, O. Groussin, F. d. r. Merlin, R. E. Milliken, and M. F. A'Hearn, "Temporal and Spatial Variability of Lunar Hydration as Observed by the Deep Impact Spacecraft", Science, 326, 565-578, 2009.

[10] Clark, R., "Detection of Adsorbed Water and Hydroxyl on the Moon", Science, 326, 562-564, 2009.

[11] McCord, T. B., L. A. Taylor, J.-P. Combe, G. Kramer, C. M. Pieters, J. M. Sunshine, and R. N. Clark, "Sources and physical processes responsible for $\mathrm{OH} / \mathrm{H} 2 \mathrm{O}$ in the lunar soil as revealed by the Moon Mineralogy Mapper (M3)", Journal of Geophysical Research, 116, 2011.

[12] Milliken, R. and S. Li, "Remote detection of widespread indigenous water in lunar pyroclastic deposits", Nature Geoscience, 10, 1038, 2017.

[13] Clark, P.E., R. MacDowall, W. Farrell, C. Brambora, T. Hurford, D. Reuter, E. Mentzell, D. Patel, S. Banks, D. Folta, N. Petro, B. Malphrus, K. Brown, "BIRCHES and Lunarcubes: Building the First Deep Space Cubesat Broadband IR Spectrometer", SPIE, 9978, 2017.

[14] Reuter, D., A. Stern, J. Scherrer, D. Jennings, J. Baer, J. Haney, L. Hardaway, A. Lunsford, S. McMuldroch, J. Moore, C. Olkin, R. Parizek, H. Reitsma, D. Sabatke, J. Spencer, J> Stone, H. Throop, J. VanCleve, G. Weigle, L. Young, "Hyperspectral sensing using the Linear Etalon Imaging Spectral Array," SPIE Proceedings, 2597, Advanced and Next-generation Satellites II, 154 (1997).

[15] Reuter, D., A. Stern, J. Baer, L. Hardaway, D. Jennings, S. McMuldroch, J. Moore, C. Olkin, R. Parizek, D. Sabatke, J. Scherrer, J. Stone, J. VanCleve, L. Young, "Ralph: A visible/infrared imager for the New Horizons Pluto/Kuiper Belt Mission", SPIE Proceedings of the Optics and Photonics Conference, Astrobiology and Planetary Missions, 5906-51, 59061F-1 to 59061F-11, 5906-51 (2005).

[16] Reuter, D., A. Stern, J. Scherrer, D. Jennings, J. Baer, J. Hanley, L. Hardaway, A. Lunsford, S. McMuldroch, J. Moore, C. Olkin, R. Parizek, H. Reitsma, D. Sabatke, J. Spencer, J. Stone, H. Throop, J. VanCleve, G. Weigle, L. Young, 
"Ralph: A visible/infrared imager for the New Horizons Pluto/Kuiper Belt Mission," Space Science Reviews, 140, 14, 129-154 (2008

[17] Reuter, D. and A. Simon-Miller, "The OVIRS Visible/IR Spectrometer on the OSIRIS-REx Mission," Lunar and Planetary Science, 1074.pdf (2012)

[18] Hardgrove, C., J. Bell, J. Thangavelautham, A. Klesh, R. Starr, T. Colaprete, M. Robinson, D. Drake, E. Johnson, J. Christian, A. Genova, D. Dunham, B. Williams, D. Nelson, A. Babuscia, P. Scowen, K. Cheung, T. McKinney, A. Taits, V. Hernandez, P. Wren, A. Thoesen, A. Godber, M. Beasley, "The Lunar Polar Hydrogen Mapper (LunaHMap) Mission: Mapping Hydrogen distributions in permanently shadowed regions on the Moons' South Pole, Lunar Exploration Analysis Group Proceedings, October, Columbia, MD, 2017.

[19] Vinckier, Q., K. Crabtree, C. Paine, P. Hayne, G. Sellar, "Design and characterization of a low cost CubesSat multiband optical receiver to map water ice on the lunar surface for the Lunar Flashlight mission", Proc of SPIE, 10403, 2017.

[20] Hardgrove, H., J. Bell, J. Thanka, A. Klesh, R. Starr, T. Colaprete, M. Robinson, D. Drake, E. Johnson, J. Christian, A. Genova, D. Dunham, B. Williams, D. Nelson, A. Babuscia, P.Scowen, K. Cheung, M. Beasley, T. McKinney, A. Taits, V. Hernandez, P. Wren, A. Thoesen, A. Godber "LunaH-Map Cubesat" AIAA Proceedings (2017)

[21] Cohen, B. "Lunar Flashlight and Other Lunar Cubesats", 2nd Planetary Cubesat Science Symposium, NASA GSFC, 2017.

[22] Clark, PE., D. Bugby, K. Chin, "Low-cost distributed networks for lunar geophysical or environmental monitoring", Lunar and Planetary Science XLIX, 2083.pdf, 2018.

[23] Clark, P.E. "Cubesats in Cislunar Space" Proc 32 ${ }^{\text {nd }}$ Annual AIAA/USU Conf Small Satellites, SSC18-V-03 (2018) 